\title{
An Immense Detail of miRNA Role in Gene Regulation and Their Expression on Functional Bases in Various Crops
}

\author{
${\text { Junaid } \text { Ali }^{1 *} \text {, Muhammad Umer Khan }}^{2}$, Hasham Feroz Ghuman ${ }^{1}$, Fahad Majeed ${ }^{4}$, Bebagar ${ }^{4}$, Ahmed Khan ${ }^{2}$, Muhammad \\ Uzair $^{2}$, Ijaz Ahmed ${ }^{4}$, Muhammad Ghani ${ }^{3}$ \\ ${ }^{1}$ Centre of Agricultural Biochemistry and Biotechnology (CABB), Pakistan \\ ${ }^{2}$ Department of Plant Breeding and Genetics, Pakistan \\ ${ }^{3}$ Department of Plant Pathology, University of Agriculture, Faisalabad-Pakistan \\ ${ }^{4}$ Department of Plant Breeding and Genetics, Faculty of Agriculture, Lasbela University of Agriculture, Water \& Marine Sciences, Uthal, Las Bela, \\ Balochistan, Pakistan
}

DOI: $10.36348 / \mathrm{sj} 1 \mathrm{~s} .2020 . \mathrm{v} 05 \mathrm{i} 07.002$

| Received: 29.06.2020 | Accepted: 08.07.2020 | Published: 26.07.2020

*Corresponding author: Junaid Ali

\section{Abstract}

Crops are very important in order to accommodate world population for food as well as industrial purposes. MiRNA (micro-RNA) have been considered as an important and crucial factor for manipulation of crops to make them more productive and resistant against harsh environmental stressed conditions. Now, these non-coding special sequences have been used successfully for gene-expression and regulation such as gene integration, slicing, signal transduction, pre and post translational modification, boost up metabolic pathways, enhancement of crop growth and developments and much more traits which is significant contribution in genetic engineering technologies for crop modifications. Genomic expression factors have been modulated through unique-miRNAs sequence which takes up toward the next generation specific targets that would have been adapted under biotechnological mechanisms and then these technologies could be used for improving the agronomic traits of various crops further down collection of high productive results. Significant strategies have pointed out to overcome the drawbacks during crop-manipulation. In this review, work for diversified and recently identified sequences of micro-RNA is studied; and production of valuable crops in order to have better agronomic properties to fulfill the requirements of food and industry with significant sustainability under stressed conditions. Genetic regulation as well as expression through miRNAs in genetic engineering will facilitate better for crop modification with quality and would be considered as positive step toward improving the economy of country.

Keywords: Non-coding sequences, crop manipulation, genomic expression, genetic regulation and environmental stresses.

Copyright @ 2020: This is an open-access article distributed under the terms of the Creative Commons Attribution license which permits unrestricted use, distribution, and reproduction in any medium for non-commercial use (NonCommercial, or CC-BY-NC) provided the original author and source are credited.

\section{INTRODUCTION}

A cell is the basic unit of life and it has the most complicated types of mechanisms which are performed by variants proteins and short sequences. The miRNA has a critical role in cleavage activity of the mRNA after transcription for regulation of the expression of the genes. It has a very short but highly conserved sequence like 20 to 24 nucleotide base pairs and theses bases are non-coding in nature, but work is being processed for inhibition of targeted transcripts by degradation of the post transcription or by translational expression. Due to its very small size researchers counted its value in different types of stresses might be biotic as well as abiotic $[1,2]$.
Recent researches show that the plants having miRNA which act as regulators of gene expression at the post transcriptional level can impaired growth and development of plant under stress condition. The miRNA constitutes on coding endogenous RNA which are comprised of rare nucleotides which regulate the expression of genes by methylation of DNA, cleavage of mRNA, translational repression and remodeling of chromosome. There are many other short sequences present in cell (small RNAs) and it is found in everywhere of cell structure usually in cytoplasm. The miRNA mainly plays an important role for massive amount of the biological processes like; the development of plant, maintenance of the genome stability, metabolism activity and enhanced or stable adaptive response in harsh environmental conditions (biotic and abiotic). By using these short sequences, we 
can manage the expression of required gene which may cause imbalance in plants. Day by day increasing population demands for sustainable food productivity which may fulfill the health requirements and for this, crops must be manipulated or improved in way to enhance the yielding as well as resistance against diseases. This will help in increasing crop yield and make them important agronomically [3].

This short sequence miRNA has a very important role as drought-resistant factor in rice. In the last few years various type of miRNA (miR170, miR172, miR408, miR1030, miR1035 etc.) evolved which control the biotic as well as abiotic and contributed to up and down regulation effects in rice [4]. This silencing technique is very effective against the sensing of nutrients and role in their enhancements and a big concerned with heavy metal detection and its removal to protect the plant life. In metals, deficiency level of cadmium as well as sulphate is detected in Brassica napus (rapeseed) and miRNA clones are made and other 5 new miRNA short sequences clones are also design which shows their effectiveness [5-7]. First time its detection was made in Arabidopsis and then carried out microarray technique to identify its role and meanwhile there were various types found there, which was controlling many factors in them (i.e. biotic as well as abiotic stresses, resistance against diseases, maintenance of the genome stability, metabolism etc) [8].

Gene for salt tolerance into maize crop (in roots) is another big achievement by using miRNA short sequence silencing technique on post transcriptional level of gene expression which greatly responses as abiotic stresses. In this finding of salt tolerance of maize inbred lines are obtained by using microfluidic array technique and the results are shown to be very effective [9]. The processes of miRNA that mostly discussed in various published research articles, are development of plant leaf, transition phase, flowering and the genome maintenance and all these are regulated through different types of miRNAs [10-14]. Although many related techniques present to manipulate the crops, but miRNA is now widely concern of researchers because its precise nature of gene silencing may help them nicely. Our precise nature of research will also handle the salt tolerance problem in wheat which may contribute in low productivity. Until now there is no reported work for wheat salinity and there is no sequencing of miRNA present related to this. MicroRNA plays an important role in biotic as well as abiotic stresses, so we can also use this into solution of salinity problem in wheat [15].

Specific classes of short non-coding sequences (miRNA) play a vital role in plant's life. These short non-coding sequences silent the various characters of unusual gene which may can cause any change into plant's behavior. The changes may be related to the plants such as integration of plant genome, resistance against biotic as well as abiotic, controlling of gene expression etc. It is the technique which deals with degradation of post-transcriptional or translational expression of mRNA. By modulation of mRNA with miRNA the productivity as well as gene expressions are changed, and it is emerging field of research that can be used in the next generation as well as genetic engineering technology to facilitate the world population [16].

In the world, population fight for shelter, food and other necessities but during last few decades this fight becomes the big issue for resources. The population demands for basic necessities and for this, new emerging technologies must be evolved that control the stresses of plants which may be biotic as well abiotic to produce the higher yield of crops. Now the miRNA is a emerging technique which protect the plant's life from various aspects by modulation of mRNA on post-transcriptional or translational expression level and this controls the phenotypic characters of gene in different environmental conditions $[17,18,16]$. MicroRNA improves the crops that are agronomically and economically very important. MIR gene (MicroRNA gene) localization is in the introns of gene as well as in the intergenic and sense or antisense orientation within a gene $[2,19]$.

There are various research projects related to locate and find out the miRNAs in a cell, but a computational approach has key role in their findings. The developing technique such as HTS (HighThroughput Sequencing) has made easy to locate the $M I R$ gene and help in to understand about their mode of action [1]. Where the miRNA works for controlling biotic and abiotic facts then there is also work reported for heavy metal scheming which enhance the crop yield in a sensible manner. To specify the research against metal deficiencies are measured during plant growth in Brassica napus (rapeseed). Cadmium and sulphate are taken as a quality control for measuring their effects with respect to deficiency and checked out the rate of plant growth during experiments. Through this experiment 5 new types of miRNA identified which is the revolutionary aspect of science. These types of short sequences are then isolated and sequenced properly then made a library for future experimentation [20].

Similarly in other crops like emmer wheat, which is the wild type, miRNA inhibition technique used because this type of wheat responds to abiotic stress such as drought? Drought-resistant genotype of wheat is produced by optimization of two mainly miRNA that are 205 and 438 in numbers. Despite these two types 13 others are also included in the drought resistant expression but have minor role as compared to 205 and 438 miRNAs. The final investigation is confirmed by qRT-PCR and the target transcripts are regulated by computationally prediction [21]. 
Abiotic stresses affect the plant because of their sessile nature. Abiotic stresses affect more than $50 \%$ yield of plants which can increase up to $70 \%$ in the coming era. These environmental fluctuations escort various metabolic and physiological changes in plants which ultimately affect their productivity and growth. Abiotic stresses can be salt stress, water stress, and, high temperature. But most important is salt stress and plants have evolved inherent mechanism to cope up salt stress plants conduct changes in the expression of gene profiles of those genes; which intricate changes in the cellular, biochemical and physiological processes such as photosynthesis, transcription, signal transduction, protein synthesis and decay [22, 23].

There are about 35 different abiotic stresses characterized into 11 groups; which are drought, wind, heat, and radiation, and oxidative stress, nutrient destitute in plants, flooding, light and toxicity of heavy metals. With the increase in population there is immense increase in the demand of food. Plants can protect themselves by altering the expression of large number of genes either by translational or transcriptional regulations. Plants works for negative regulators by down regulating them and for protective genes they act as up regulators [24-26].

Recently, to control different abiotic stresses against plants several genes which encode protein are being identified, but we have limited knowledge about their regulatory mechanism. To re-gain and re-build cellular homeostasis of plants; these post transcriptional activities are important [27, 28]. These miRNAs are different from each other in their biogenesis, size, regulatory role and mode of action and are found abundantly in plants and by base binding with the transcription factors which are the targets of complementary mRNA played major role in the post transcriptional regulations [29, 30].

These miRNAs found either in the intergenic areas or in the antisense (or sense) orientation within the gene's introns.' In the genome some miRNA can also be found as cluster shape or can exist as long polycistronic RNA's which are transcribed together. They are mainly produced in the nucleus, but some specific miRNAs are transcribed into long primary transcripts by RNA polymerase II. These miRNAs are capped with specially nucleotides which are modified at 5 ' end and at 3' end they are polyadenylated with the adenosines. The lit-7 and lin-4 regulatory RNA's are identified as the precursors and conserved sequences, and these are conserved across animal and plant species. As there is no conservation occurs between plants and animal, but miRNA shows high conservation [31-33].

Microarray technology; that has been practiced in the elevated throughput finding of gene appearance or expression and it has been demonstrated a helpfulinstrument; in miRNA expression assays [34]. The microarray-basedexploration was carried to find out the sub-mergence and stress responsive miRNAs into the maize roots [35]. The high level of salinity; is a severe abiotic pressure (stress) for maize seedlings and micro-RNA based guided post transcriptional regulation; may be concerned into the response through salt stunned maize seedlings. The study on maize has been pointed out that; only a small number of diversify expressed micro-RNA's which wasevenly regulated among2 maize lines; and in them the vast bulk of the diversearticulated micro-RNA's demonstratethe genotype specific look (expression) model. These expressed and regulated miRNA's actively play critical as well as vital roles into morphological (phenotypic characters) or metabolic adjustmentreaction in the salt shocked root cells of maize and a genotypespecific appearance (expression) model might be explain the significant salt sensitivities among2 maize lines $[25,16$, $34,36,15]$.

A protein expression coding for genes, is synchronized at both levels i.e. transcriptional as well as post transcriptional and this might be due to through avariety of categories of molecules. With respect to cisregulatoryas well as trans-regulatory DNA sequences, and various sorts of Trans acting proteins recently,small RNA molecules have been come out as significant regulatory molecules into the eukaryotic cells [31, 27]. The regulation through short or small RNA's can be result out into both transcriptional as well as posttranscriptional repression of the gene expression. In the plants, these short RNA's reveal an un-predictable complexity and they have been classified based on their biogenesis as well as the structure of genomic loci through which these are transcribed. Majority of the micro-RNA types might be increased into the near future like the outcome of the comprehensive analysis of the large extent of micro-RNA sequencing projects. Meanwhile, there is distinguish among micro-RNA's (miRNAs) and 3types of the endogenous short or small interfering RNAs (siRNAs) i.e. Trans acting siRNA (tasiRNA), heterochromatic siRNA (hc-siRNA) and natural antisense siRNA (nat-siRNA). And all these have expressed their own characters on the bases of natural extension [37-40].

\section{Gene-Regulation and Gene-Expression by Micro- RNA}

The small-RNA are very important to regulate all necessary pathways in order to maintain the various traits in plants by TFs (transcription factors); while sRNAs almost have specific matches in genome that is RNA-encoded in fact. Nowadays, micro-RNA used for gene-regulations as well as expression of genes which basically being investigated regularly worldwide [41$44,16]$. In plant biology, it has a great impact for regulation of genes and expression; while it consists of almost 20-24 nucleotide base pairs in length and regulated all traits by post transcriptional phase that 
show high degree of complementarity. In later studies, the targeting points oh miRNA are related to target one which shows the relatedness for traits and control them at translation expression. Its activity done by the removal of tail (poly-A tail) from mRNA and finally destabilize it for further expressions [44, 45].

Meanwhile, miRNA plays very impressive role to control robustness of the biological progressions due to perform activity of silencing of transcriptional factors and suppress arbitrary fluctuations into transcript-copy numbers [46]. Various length-based miRNA can be generated through same genes or from same genetic-pool which might be heterogenous in nature [47]. DCL-1 gene in plants is responsible to generate the miRNA having 21 nucleotide bases meanwhile, DCL-2, DCL-3, and DCL-4 are also involved in generating miRNA with variable lengths [48]. The variation genetics of miRNA with multiple lengths in cell mainly involves in producing practical based-cassettes (based on precursor expressions) which may help in creating artificially specific-sequences containing miRNA that could help in plant improvements as well as developments [47].

\section{Mechanisms for Targets-Screening and Predictions of Micro-RNA}

In the targeting of specific genes by miRNA have been predicting due to bioinformatics-based tools which almost shows experimental approaches [43, 49, 50]. Some bioinformatics-based tools have been used for miRNA-targeting predictions on the basis of scoring (complementary scoring) as well as secondary structures investigations such as TAPIR, miRTarBase, miRTour, and psRNATarget are included in database (validated miRNA target interactions database) on experimental features at commercial level [51, [50]. Meanwhile, the targets of miRNA can be evaluated or monitored due to RT-PCR and cutting sites of the targets have been mapped by RACE (5'-RapidAmplification of cDNA Ends). In the recent time, another modified process of analyzing sequences (degradome sequencing technique) was reported which is basically considered as improved/enhanced version of 5'-Rapid-Amplification of cDNA Ends (RACE) that has better quality for data analyzing with deep learning/sequencing mechanism and shows high throughput results [52, 53, 49]. This sequencing (degradome sequencing) method have been used to optimize cleaved target-points on the bases of relative abundance [49].

\section{Modification in crops through miRNAs-sequence with suitable approaches Oryza Sativa (Rice)}

Rice is considered as the largest as well as vital source for food worldwide. Rice crop also has some serious challenges (regarding environmental stresses i.e. cold, salinity, drought, heat, and deficiency of nutrients) just like other cereal crops have.
Meanwhile, there are nearly 20 different types of microRNA were isolated from the preserve rice-library (Rice cDNA-Library). From rice cDNA-Library, potential gene targets were identified such as miR-5 (Metabolism), miR-10 (Resistance for Diseases), miR-4 and miR-15 (Transportation), miR-4 (Transcription) etc. In the same year, other almost 35 miRNAs were identified in rice; among them 14 miRNAs were considered as very novel that show specific target-genes which were characterized for numerous physiological mechanisms for rice $[54,55]$.

Nowadays in rice, numerous miRNAs sequences (592-miRNAs sequences) have been discovered and can be found at miRbase site (version21). A big data collection can be found to dig out the information regarding miRNAs identification in rice through NGS data and various micro-RNAs have been known to show response against abiotic stresses [5658]. In order to response against for high temperature (heat or cold), there were targeted genes of miRNAs (miR-1425, miR-319, miR-167 and miR-812q) isolated which show better withstand quality under very high range of temperature (cold stress) that make the rice more productive and valuable [59]. While OsmiR-397 have been reported to show response against high temperature stress (heat stress) by special modulation (L-ascorbate oxidase activity) in rice which make it more susceptible to environment [59, 60]. It has been concluded that if OsmiR-397 secreted excessively (over expression) then downregulation of the L-ascorbate oxidase (targeted genes) might be involved in high yields production as well as increases the grain size remarkably and improve the panicle branching [61]. Recent studies showed the better result of rice against different variables such as OsamiR820 over-expression give nice response against heat, light, drought, salinitybased stresses; while OsamiR7695 shows the high resistance for fungal diseases or pathogens $[62,63]$.

\section{Zea mays (Maize)}

Maize considered as very important crop worldwide. It is believed second significant crop which utilized for feed, food, forage as well as being used to produce ethanol. As whole genome sequence of maize has been done, this crop is used as model crop for research purposes [64]. There are 5 different types of micro-RNAs (miR156, miR160, miR166, miR167, miR169) reported which integrated in biotic and abiotic stresses; despite stresses, these miRNAs are also involved in germination, development, growth, and sustainability of plants [65]. In addition to stresses as well as growth and development with respect to maize plants, some miRbase-versions (21-lists 321 miRNAs) play very important and crucial role in it. previous studies revealed that miR-164 is actively participated in negative regulation of ZmNACI (including CUC, ATAF, NAM), a precise plant TF-family which tangled in stress and development regulation of plants [66]. Regulatory role during the development of endosperm 
in maize, mRNAs (95-conserved mRNAs: miR156a, miR160a, miR164e, miR164a, miR167d, miR168, miR169a, miR393a, miR397b, miR408b, miR528a etc. related to 20 families) and other eleven microRNA families have been involved in this function [67].

Moreover, maize plants demonstrate 3 miRNAs such as mir160b, miR167a and mir528a (regulators of auxin response factor and a member of the B3 TF family, and regulator of a putative laccase, a Ring-H2 zinc finger protein and a MADS box-like protein) which are considered as very critical for plantphysiology, development as well as ear germination [68]. In recent researches, some maize microRNAs (124-conserved and 68 novel miRNAs) have been recognized that specifically associated in response to stress such as drought, where they thought to be regulation of gene for drought-stress [31].

\section{Triticum aestivum L. (Wheat)}

Among cereal crop, wheat has its own value and considered as the chief crop in order to minimize hunger as well as nutritional values worldwide. It has a critical importance in compensation or contribution to food security. There is large data regarding wheat crop available for researching; while in this aspect, some miRNAs have been investigated in wheat that are very helpful in plant physiology and anatomy. Almost 23novel (miR501, miR502, miR 503, miR504, miR505 miR506, miR510, miR514, and miR516, etc.; Yao et al., [69] and 58 other conserved-miRNAs (consist of 43 families) (miR160, miR164, miR165/166, miR167, miR168, miR169, miR170/171, miR172, miR319, miR390, miR393, miR396, miR397, miR399, and miR408,) and 23 were novel (miR501, miR502, miR503, miR504 etc.) were investigated during studies which regulate plant growth, developments, and germination with stability. These miRNAs sequences have been used to localize the specific target genes (APETELA2 protein, MYB, MADS box protein, homeodomain-leucine zipper protein, squamosa promoter binding proteins, CCAAT-binding protein, NAC1, blue copper protein, scarecrow-like protein, and auxin response factor) in wheat that actively play significant role in physiological processes, growth and developments as well as responses to biotic or abiotic stresses [69].

In miRbase (version-21), numerous microRNAs were recognized (116-miRNAs sequences). Almost 62 miRNAs (conserved) have been investigated by EST-analysis and predicted target-genes were acknowledged to be considered in numerous biological mechanisms such as miR5181, miR5175 (ion transportation), miR1436, miR1439, miR5067, miR5205 (disease resistance), miR774, miR1126 (metabolic pathways), miR395d, miR1435 (abiotic stress response), miR397, miR437 (development), as well as miR530, miR5175 (signal transduction) [70]. Recently, Genome-Wide Association Analysis investigation has been conducted regarding wheat genome, almost 323 miRNAs (novel-miRNAs) related to 276-families have been identified in association with potential targeted genes. Meanwhile, they were recognized in the grain of wheat and thought to be involved in its development and some other sequences (Ta-miR1122, Ta-miR1117, TamiR1134 and TamiR113, Ta-miR5653, Ta-miR855, Ta-miR819k, TamiR3708, Ta-miR5156) also reported that contribute towards responses for abiotic stresses [71]. Stress responses, germination, growth, development and physiological responses regulated by specific targeted potential genes which used to encode for POZ or BTB domain containing protein, Ubiquitin carrier-proteins, Kelch/Fbox repeat proteins, TFs also [72].

\section{Glycine max L. (Soybean)}

Like cereal crop, soybean also has its own importance with respect to oil contents. This crop is known for oil production and used to take heavy carbohydrates through this. Usually it contains $20 \%$ heavy oil contents while $40 \%$ protein contents which equally important for humans as well as animals. Research regarding miRNA has been started in 2008 when almost 20 conserved miRNAs (miR393, miR169, miR156, miR164, etc.) related to 55 families and some new miRNAs (35 miRNAs; miR1512, miR1507, miR1515, miR482, miR1511, etc.) were investigated in soybean [73]. Now successfully, almost 693 sequences (soybean-miRNAs) have been submitted to database (miRbase version 21). There were 26 sequences of miRNAs recognized which involved for the development of seeds as well as their predicted potential-targets such as ARF, TCP-type, TFs, NAC, MYB and GRF families. And some other genes (nonconserved) like protein suppressor (gene-silencing 3), G-protein, and F-box protein also included in it [52].

Such type of researching points (reports) show the regulatory association of different sequences of miRNAs that define the intended functions of them into soybean seed developments. Moreover, soybean has the remarkable abilities to tolerate numerous environmental stresses such as salinity, drought, temperature and having susceptibility to these stresses as well. But in many other investigations, soybean susceptibility has been improved by focusing on the regulation of miRNA-sequences. Reported work indicated the drought tolerance in soybean roots due to gene regulation of small RNA-sequences [74]. While other numerous miRNAs sequences were also investigated that actively involved in metal resistance like aluminum stress in wild-type soybean [75]. Some disease related functions of miRNAs sequences have been found in soybean such as phytophthora sojae (root rot) and rust infections, which mainly affect the mature as well as seedlings of soybean plants $[76,74]$. 


\section{Gossypium hirsutum L. (Cotton)}

Cotton has important rank among crops worldwide due to its value-added nature towards edible oil, fiber, lint, as well as textile product. Despite numerous values, this crop is taken up as a model crop for variable traits such as cellulose and cell wall biosynthesis and polyploidization in plants. Also, cotton is considered as $5^{\text {th }}$ largest crop for oil production and $2^{\text {nd }}$ most significant crop for protein resources [77]. Identification and characterization of miRNAs in cotton, first reported in 2007, and multiple miRNAsequences (hundreds of sequences like 158 almost added to miRbase version 21 database) evolved with variable traits [78, 79]. The mentioned sequences of miRNA were play important role in order to regulate the metabolic as well as biological pathways such as germination, development (floral development also), growth, fiber initialization, and improved response to stresses (biotic and abiotic) or pathogens [78, 80]. Zhang et al., [78], findings revealed that various miRNAs like miR782, miR414 and miR396 based proteins (fiber-protein fb23, callose synthase, and quinone oxido-reductase) put significant role in fiber development and its differentiation. Seventeen (17) miRNAs (related to 8 families) were identified that provided tolerance to cotton against salinity and responded effectively in salt tolerant cultivars than susceptible [81].

Recent advances in studies showed that various miRNAs have very positive effect on cotton in order to response against metal-stress. This metal stress tolerance in cotton have significant changes in plant's organs like change in photosynthesis patterns, chloroplast ultrastructure, damaging of cell membrane induction, and growth-inhibition [82]. Various other miRNA-sequences have been reported that play substantial role against insect-pest attack, defense against diseases and its invasions. For instance, according to recent investigations, 58 conserved miRNA sequences (related to 140 families) were tangled in response against verticillium wilt (vascular disease that mainly damage development and growth of cotton) due to regulation of defensive pathway which was considered as important tool to make it susceptible against numerous diseases [83].

\section{Improvement in crops and Tolerance to stresses (biotic and abiotic) due to mRNA-strategies}

The behavior of plants towards stress responses (biotic or abiotic) totally depends upon their molecular pathways as well as their inter-relationships to each other and some other small sequences like miRNA-sequences also involved in it [18]. Moreover, resistance against diseases and active-positive responses for stresses in crops lead for their stability and susceptibility towards enhancement of quality yield. There is an important investigation indicated that tomato plants show the decrement in opening of stomata (this shows the lower level of transpiration in plants which mainly effective in water loss conditions) and having improved response against drought just because of the miR169 expression [53]. In case of rice, alkali and salt stresses tolerance have been investigated due to regulation of osa-miR396c gene in stress conditions and increase its susceptibility [84]. In the same way, transgenic rice having gene miR398 showed more resistance against increased drought and salinity than non-transgenic [85]. While the better or more expression of miR319 gene in rice was noticed to show morphogenesis in leaves and having good response during acclimation in chilling temperature [86].

Recently, numerous studies have been taken on miRNAs role in plants and this shows the improved response against environmental stresses such as biotic and abiotic. In rice, blast infection is considered as very harmful for yielding and quality but some regulatory miRNA sequences like osa-miR7696 have shown positive impact on it and enhanced the response in rice [62]. Meanwhile, bacterial growth and infection also controlled/inhibit due to miR393 gene which was used later to develop plant immunity against diseases [87]. Another investigation shows that bacterial growth and infection also be controlled by regulation (positively and negatively) of miR160a, miR398b, and miR377 genes which control the deposition of callose by MAMP-induction. And this may use later to investigate the regulatory function of miRNAs in plant- immunity (innate) [88]. Arabidopsis thaliana also selected (as a model plant) for investigation of role of miRNA sequences which give the better sense, but functional analysis for miRNAs sequences showed the least studies regarding translational impact. Therefore, regulation of miRNA-sequences was noticed interestingly in order to tolerate the environmental stresses (biotic and abiotic) in many crops and they play very active role in sustainability as well as susceptibility [49].

\section{miRNA-modifications by Genome-Editing Tactics}

Micro-RNAs are considered as the most valueable factors to regulate the plant functions in stress conditions. Many investigations have been made in order to attain the mechanisms for repress or over expression of miRNA genes for improvement of plants [89, 87, 90, 84, 53, 62]. According to reported data, there is a least loss have been acknowledged due to very short sequences of miRNA in crops. Technologies (two Genome editing) especially by nucleases, have been considered as important tool for manipulation in plant genome for specific traits. These technologies provide significant as well as precise nature of engineering of genome that enables crop to cope with harsh conditions. Transcription Activator Like Effector Nuclease (TALENs) and Clustered Regularly Interspaced Short Palindromic Repeats (CRISPR), these genome editing technologies are used for genomemanipulation such as insertion or deletion [91]. Among research, CRISPR/CAS9 technology has been found 
very effective and useful due to the most simple and easy structure and interestingly applicable to numerous organisms for their genome editing [92, 93]. According to another investigation, CRISPR/CAS9 with gRNA has been used for editing and cleavage of rice plantgenome. There are almost 9-endigenous loci were estimated to modify and this system (CRISPR-CAS9) also used for knockout of GFP (green fluorescent protein) transgene in soybean [94, 95].

Recently, CRISPR-CAS9 with sgRNA (single guided RNA) has been cloned which target the processing sites of biogenesis in cancer (human cancer cell lines). This targeting was approached due to specific mRNAs such as miR141, miR200c, and miR17 in human. By this genome editing technology (CRISPR-CAS9), almost $96 \%$ of the small RNAsequences have been suppressed and their expression decrement in human cancer lead to treatment for it in more accurate manners [92]. These genome manipulation technologies have been considered as revolution in the field of plants due to improvements by editing. This technology is now used in model as well as other plants such as tobacco, Arabidopsis, maize, wheat, tomato, rice, sweet orange and sorghum. But despite it, applications on the bases of genome-editing technology of some RNAs (like non-coding RNAs) is promising yet in plants [96]. CRISPR-CAS technology is very emerging as well as powerful tool which considered as pre-requisite for gene-targeting and editing for better traits; it also significant feature for the characterization of genomic pool based on functions in plants. This technology is very precise and important for manipulation of small RNA-sequences or genes by which modifications are done with improved agronomic trait of crops.

\section{CONCLUSION}

Micro-RNAs are considered as the most important and significant sequences for the regulation of genes under crucial circumstances. These short segments are being used for crop manipulation as well as to improve them against specific stresses. Valuable properties have been concerned for modifications in plants due to mi-RNAs such as maintenance of plants during homeostasis, resistance against biotic and abiotic stresses, plant growth and developments, hormonal balance and its approach to specific site, signaling pathways and changing of phases from vegetative to reproductive. Computational biology is an important tool to analyze the best way to regulate fine sequences for plan's modifications and it would also give the right future perspective to utilize them into wet-lab processing. This biological branch also indicates the expression level of gene regulators under diversified nature of short sequences which might be used to enhance the agriculturally better traits for plants that usually beneficial for them to sustain under critical conditions. It's pretty true for miRNAs that they play vital role in plant modifications as well as improvements but still some unintended drawbacks have been seen since last few years. Although, they could be used for specific gene regulation with suitable of micro-RNAs but there should be immense need to find out some other regulatory mechanisms which might be helpful in production of plants with fruitful results instead of side-effects. In this review, total RNA and other short sequences have been considered as important tool for genomic expression as well as regulation that could be productive for crop improvements and show higher level of resistance against environmental stresses. We have summarized some important and highly motivated facts for cropmanagement under stressed-conditions. Agronomically engineered crops could be considered as contribution towards country economy.

\section{REFERENCES}

1. Tripathi, A., Goswami, K., \& Sanan-Mishra, N. (2015). Role of bioinformatics in establishing microRNAs as modulators of abiotic stress responses: the new revolution. Frontiers in Physiology, 6, 286.

2. Bartel, D. P. (2004). MicroRNAs: genomics, biogenesis, mechanism, and function. cell, 116(2), 281-297.

3. Djami-Tchatchou, A. T., Sanan-Mishra, N., Ntushelo, K., \& Dubery, I. A. (2017). Functional roles of microRNAs in agronomically important plants - potential as targets for crop improvement and protection. Frontiers in plant science, 8, 378.

4. Li, W. X., Oono, Y., Zhu, J., He, X. J., Wu, J. M., Iida, K., ... \& Zhu, J. K. (2008). The Arabidopsis NFYA5 transcription factor is regulated transcriptionally and posttranscriptionally to promote drought resistance. The Plant Cell, 20(8), 2238-2251.

5. Tang, S., Wang, Y., Li, Z., Gui, Y., Xiao, B., Xie, J., ... \& Fan, L. (2012). Identification of wounding and topping responsive small RNAs in tobacco (Nicotiana tabacum). BMC plant biology, 12(1), 28.

6. Zhao, B., Ge, L., Liang, R., Li, W., Ruan, K., Lin, H., \& Jin, Y. (2009). Members of miR-169 family are induced by high salinity and transiently inhibit the NF-YA transcription factor. BMC molecular biology, 10(1), 29.

7. Shi, Z. M., Wang, J., Yan, Z., You, Y. P., Li, C. Y., Qian, X., ... \& Li, M. N. (2012). MiR-128 inhibits tumor growth and angiogenesis by targeting p70S6K1. PloS one, 7(3), e32709.

8. Yin, Z., Li, Y., Yu, J., Liu, Y., Li, C., Han, X., \& Shen, F. (2012). Difference in miRNA expression profiles between two cotton cultivars with distinct salt sensitivity. Molecular biology reports, 39(4), 4961-4970.

9. Ding, J., Zhou, S., \& Guan, J. (2012). Finding microRNA targets in plants: current status and perspectives. Genomics, proteomics \& bioinformatics, 10(5), 264-275. 
10. Aukerman, M. J., \& Sakai, H. (2003). Regulation of flowering time and floral organ identity by a microRNA and its APETALA2-like target genes. The Plant Cell, 15(11), 2730-2741.

11. Palatnik, J. F., Allen, E., Wu, X., Schommer, C., Schwab, R., Carrington, J. C., \& Weigel, D. (2003). Control of leaf morphogenesis by microRNAs. Nature, 425(6955), 257-263.

12. Vaucheret, H. (2009). AGO1 homeostasis involves differential production of 21-nt and 22-nt miR168 species by MIR168a and MIR168b. PLoS One. 4:e6442.

13. Guo, H. S., Xie, Q., Fei, J. F., \& Chua, N. H. (2005). MicroRNA directs mRNA cleavage of the transcription factor NAC1 to downregulate auxin signals for Arabidopsis lateral root development. The Plant Cell, 17(5), 1376-1386.

14. Mallory, A. C., \& Vaucheret, H. (2006). Functions of microRNAs and related small RNAs in plants. Nat Genet. 38(Suppl.), S31-S36.

15. Ryan, P. R., Tyerman, S. D., Sasaki, T., Furuichi, T., Yamamoto, Y., Zhang, W. H., \& Delhaize, E. (2011). The identification of aluminium-resistance genes provides opportunities for enhancing crop production on acid soils. Journal of Experimental Botany, 62(1), 9-20.

16. Djami-Tchatchou, A. T., \& Dubery, I. A. (2015). Lipopolysaccharide perception leads to dynamic alterations in the microtranscriptome of Arabidopsis thaliana cells and leaf tissues. $B M C$ plant biology, 15(1), 79.

17. Sanan-Mishra, N., Kumar, V., Sopory, S. K., \& Mukherjee, S. K. (2009). Cloning and validation of novel miRNA from basmati rice indicates cross talk between abiotic and biotic stresses. Molecular Genetics and Genomics, 282(5), 463.

18. Bej, S., \& Basak, J. (2014). MicroRNAs: the potential biomarkers in plant stress response. American Journal of Plant Sciences, 2014. Am J Plant Sci. 5:748-759.

19. Bartel, J. R. M. B. D. (1953). B. 2006 MicroRNAs and their regulatory roles in plants. Annu. Rev. Plant Biol, 57, 19-53.

20. Hsu, S. D., Lin, F. M., Wu, W. Y., Liang, C., Huang, W. C., Chan, W. L., ... \& Chien, C. H. (2011). miRTarBase: a database curates experimentally validated microRNA-target interactions. Nucleic acids research,39(suppl_1), D163-D169.

21. Kantar, M., Lucas, S. J., \& Budak, H. (2011). miRNA expression patterns of Triticum dicoccoides in response to shock drought stress. Planta, 233(3), 471-484.

22. Urano, K., Delrot, S., Pezzotti, M., \& Shinozaki, K. (2011). Effects of abiotic stress on plants: a systems biology perspective. BMC Plant Biol. 11:163.

23. Ghosh, D., \& Xu, J. (2014). Abiotic stress responses in plant roots: a proteomics perspective. Frontiers in plant science, 5, 6.
24. Mahajan, S., \& Tuteja, N. (2005). Cold, salinity and drought stresses: an overview. Archives of biochemistry and biophysics, 444(2), 139-158.

25. Nakashima, K., Ito, Y., \& Yamaguchi-Shinozaki, K. (2009). Transcriptional regulatory networks in response to abiotic stresses in Arabidopsis and grasses. Plant physiology, 149(1), 88-95.

26. Pfalz, J., Liebers, M., Hirth, M., Grübler, B., Holtzegel, U., Schröter, Y., ... \& Pfannschmidt, T. (2012). Environmental control of plant nuclear gene expression by chloroplast redox signals. Frontiers in Plant Science, 3, 257.

27. de Oliveira, A. B., Alencar, N. L. M., \& GomesFilho, E. (2013). Comparison between the water and salt stress effects on plant growth and development. Responses of organisms to water stress, 67.

28. Kurihara, Y., Takashi, Y., \& Watanabe, Y. (2006). The interaction between DCL1 and HYL1 is important for efficient and precise processing of pri-miRNA in plant microRNA biogenesis. Rna, 12(2), 206-212.

29. NOBEL, P. S., \& CUI, M. (1992). Hydraulic conductances of the soil, the root-soil air gap, and the root: changes for desert succulents in drying soil. Journal of Experimental Botany, 43(3), 319326.

30. Aroca, R., Porcel, R., \& Ruiz-Lozano, J. M. (2012). Regulation of root water uptake under abiotic stress conditions. Journal of experimental botany, 63(1), 43-57.

31. Sheng, L., Chai, W., Gong, X., Zhou, L., Cai, R., Li, X., ... \& Cheng, B. (2015). Identification and characterization of novel maize miRNAs involved in different genetic background. International journal of biological sciences, 11(7), 781-793.

32. Prabu, G. R., \& Mandal, A. K. (2010). Computational identification of miRNAs and their target genes from expressed sequence tags of tea (Camellia sinensis). Genom Proteom Bioinform 8 (2): 113-121.

33. Eamens, A. L., \& Wang, M. B. (2011). Alternate approaches to repress endogenous microRNA activity in Arabidopsis thaliana. Plant signaling \& behavior, 6(3), 349-359.

34. Liu, X., Hua, X., Guo, J., Qi, D., Wang, L., Liu, Z., ... \& Liu, G. (2008). Enhanced tolerance to drought stress in transgenic tobacco plants overexpressing VTE1 for increased tocopherol production from Arabidopsis thaliana. Biotechnology letters, 30(7), 1275-1280.

35. Zhang, Z., Yu, J., Li, D., Zhang, Z., Liu, F., Zhou, X., ... \& Su, Z. (2010). PMRD: plant microRNA database. Nucleic acids research,38(suppl_1), D806-D813.

36. Steffens, N. O., Galuschka, C., Schindler, M., Bülow, L., \& Hehl, R. (2004). AthaMap: an online resource for in silico transcription factor binding sites in the Arabidopsis thaliana genome. Nucleic acids research, 32(suppl_1), D368-D372. 
37. Ramachandran, V., \& Chen, X. (2008). Small RNA metabolism in Arabidopsis. Trends in plant science, 13(7), 368-374.

38. Jamalkandi, S. A., \& Masoudi-Nejad, A. (2009). Reconstruction of Arabidopsis thaliana fully integrated small RNA pathway. Functional \& integrative genomics, 9(4), 419-432.

39. Liu, Q., \& Paroo, Z. (2010). Biochemical principles of small RNA pathways. Annual review of biochemistry, 79, 295-319.

40. Voinnet, O. (2009). Origin, biogenesis, and activity of plant microRNAs. Cell, 136(4), 669687.

41. Mishra, N. S., \& Mukherjee, S. K. (2007). A peep into the plant miRNA world. The Open Plant Science Journal, 1(1):1-9.

42. Lelandais-Brière, C., Sorin, C., Declerck, M., Benslimane, A., Crespi, M., \& Hartmann, C. (2010). Small RNA diversity in plants and its impact in development. Current genomics, 11(1), 14-23.

43. Pantaleo, V., Szittya, G., Moxon, S., Miozzi, L., Moulton, V., Dalmay, T., \& Burgyan, J. (2010). Identification of grapevine microRNAs and their targets using high-throughput sequencing and degradome analysis. The Plant Journal, 62(6), 960-976.

44. Sun, G., Stewart, C. N., Xiao, P., \& Zhang, B. (2012). MicroRNA Expression Analysis in the Cellulosic Biofuel Crop Switchgrass () under Abiotic Stress. PLoS ONE. 7:e32017.

45. Guleria, P., Mahajan, M., Bhardwaj, J., \& Yadav, S. K. (2011). Plant small RNAs: biogenesis, mode of action and their roles in abiotic stresses. Genomics, proteomics \& bioinformatics, 9(6), 183-199.

46. Ebert, M. S., \& Sharp, P. A. (2012). Roles for microRNAs in conferring robustness to biological processes. Cell, 149(3), 515-524.

47. Starega-Roslan, J., Krol, J., Koscianska, E., Kozlowski, P., Szlachcic, W. J., Sobczak, K., \& Krzyzosiak, W. J. (2011). Structural basis of microRNA length variety. Nucleic acids research, 39(1), 257-268.

48. Jeong, D. H. (2016). Functional diversity of microRNA variants in plants. Journal of Plant Biology, 59(4), 303-310.

49. Zhang, S., Shi, W., Chen, Y., Xu, Z., Zhu, J., Zhang, T., ... \& Zhang, X. (2015). Overexpression of SYF2 correlates with enhanced cell growth and poor prognosis in human hepatocellular carcinoma. Molecular and cellular biochemistry, 410(1-2), 1-9.

50. Shriram, V., Kumar, V., Devarumath, R. M., Khare, T. S., \& Wani, S. H. (2016). MicroRNAs as potential targets for abiotic stress tolerance in plants. Frontiers in Plant Science, 7, 817.

51. Hsu, S. D., Lin, F. M., Wu, W. Y., Liang, C., Huang, W. C., Chan, W. L., ... \& Chien, C. H. (2011). miRTarBase: a database curates experimentally validated microRNA-target interactions. Nucleic acids research,39(suppl_1), D163-D169.

52. Song, Q. X., Liu, Y. F., Hu, X. Y., Zhang, W. K., Ma, B., Chen, S. Y., \& Zhang, J. S. (2011). Identification of miRNAs and their target genes in developing soybean seeds by deep sequencing. BMC plant biology, 11(1), 5.

53. Zhang, B., Wang, Q., \& Pan, X. (2007). MicroRNAs and their regulatory roles in animals and plants. Journal of cellular physiology, 210(2), 279-289.

54. Wang, J. F., Zhou, H., Chen, Y. Q., Luo, Q. J., \& $\mathrm{Qu}$, L. H. (2004). Identification of 20 microRNAs from Oryza sativa. Nucleic Acids Research, 32(5), 1688-1695.

55. Sunkar, R., Girke, T., Jain, P. K., \& Zhu, J. K. (2005). Cloning and characterization of microRNAs from rice. The Plant Cell, 17(5), 1397-1411.

56. Sunkar, R., Chinnusamy, V., Zhu, J., \& Zhu, J. K. (2007). Small RNAs as big players in plant abiotic stress responses and nutrient deprivation. Trends in plant science, 12(7), 301-309.

57. Zhang, B., Pan, X., Wang, Q., Cobb, G. P., \& Anderson, T. A. (2006). Computational identification of microRNAs and their targets. Computational biology and chemistry, 30(6), 395-407.

58. Mittal, S., Rani, A., \& Mehra, R. (2016). Radon levels in drinking water and soil samples of Jodhpur and Nagaur districts of Rajasthan, India. Applied Radiation and Isotopes, 113, 53-59.

59. Jeong, D. H., Park, S., Zhai, J., Gurazada, S. G. R., De Paoli, E., \& Green, P. J. (2011). Meyers BC. Massive analysis of rice small RNAs: mechanistic implications of regulated microRNAs and variants for differential target RNA cleavage. Plant Cell, 23(12), 4185-4207.

60. Yu, X., Wang, H., Lu, Y., de Ruiter, M., Cariaso, M., Prins, M., ... \& He, Y. (2012). Identification of conserved and novel microRNAs that are responsive to heat stress in Brassica rapa. Journal of Experimental Botany, 63(2), 1025-1038.

61. Zhang, Y. C., Yu, Y., Wang, C. Y., Li, Z. Y., Liu, Q., Xu, J., ... \& Xin, P. (2013). Overexpression of microRNA OsmiR397 improves rice yield by increasing grain size and promoting panicle branching. Nature biotechnology, 31(9), 848-852.

62. Campo, S., Peris-Peris, C., Siré, C., Moreno, A. B., Donaire, L., Zytnicki, M., ... \& San Segundo, B. (2013). Identification of a novel micro RNA (mi RNA) from rice that targets an alternatively spliced transcript of the $\mathrm{N}$ ramp6 ( $\mathrm{N}$ atural resistance-associated macrophage protein 6) gene involved in pathogen resistance. New Phytologist, 199(1), 212-227.

63. Sharma, N., Panchal, S., \& Sanan-Mishra, N. (2015). Protocol for artificial microRNA mediated over-expression of miR820 in indica 
rice. American Journal of Plant Sciences, 6(12), 1951.

64. Bennetzen, J. L., \& Hake, S. C. (2009). Handbook of Maize, Its Biology. New York, NY: Springer.

65. Mica, E., Gianfranceschi, L., \& Pe, M. E. (2006). Characterization of five microRNA families in maize. Journal of experimental botany,57(11), 2601-2612.

66. Li, J., Guo, G., Guo, W., Guo, G., Tong, D., Ni, Z., ... \& Yao, Y. (2012). miRNA164-directed cleavage of ZmNAC1 confers lateral root development in maize (Zea maysL.). BMC plant biology, 12(1), 220.

67. Gu, Y., Liu, Y., Zhang, J., Liu, H., Hu, Y., Du, H., ... \& Huang, Y. (2013). Identification and characterization of microRNAs in the developing maize endosperm. Genomics, 102(5-6), 472-478.

68. Ding, H., Gao, J., Luo, M., Peng, H., Lin, H., Yuan, G., ... \& Zhang, Z. (2013). Identification and functional analysis of miRNAs in developing kernels of a viviparous mutant in maize. The Crop Journal, 1(2), 115-126.

69. Yao, Y., Guo, G., Ni, Z., Sunkar, R., Du, J., Zhu, J. K., \& Sun, Q. (2007). Cloning and characterization of microRNAs from wheat (Triticum aestivum L.). Genome biology, 8(6), R96.

70. Han, J., Kong, M. L., Xie, H., Sun, Q. P., Nan, Z. J., Zhang, Q. Z., \& Pan, J. B. (2013). Identification of miRNAs and their targets in wheat (Triticum aestivum L.) by EST analysis. Genet Mol Res, 12(3793), 805.

71. Lur, H. S., \& Setter, T. L. (1993). Role of auxin in maize endosperm development (timing of nuclear DNA endoreduplication, zein expression, and cytokinin). Plant Physiology, 103(1), 273-280.

72. Pandey, B., Gupta, O. P., Pandey, D. M., Sharma, I., \& Sharma, P. (2013). Identification of new stress-induced microRNA and their targets in wheat using computational approach. Plant signaling \& behavior, 8(5), e23932.

73. Subramanian, S., Fu, Y., Sunkar, R., Barbazuk, W. B., Zhu, J. K., \& Yu, O. (2008). Novel and nodulation-regulated microRNAs in soybean roots. BMC genomics, 9(1), 160.

74. Kulcheski, F. R., de Oliveira, L. F., Molina, L. G., Almerão, M. P., Rodrigues, F. A., Marcolino, J., ... \& Abdelnoor, R. V. (2011). Identification of novel soybean microRNAs involved in abiotic and biotic stresses. BMC genomics, 12(1), 307.

75. Zeng, Q. Y., Yang, C. Y., Ma, Q. B., Li, X. P., Dong, W. W., \& Nian, H. (2012). Identification of wild soybean miRNAs and their target genes responsive to aluminum stress. BMC Plant Biology, 12(1), 182.

76. Jingi, W. A. N. G., Liu, C. Y., Zhang, L. W., Wang, J. L., Hu, G. H., Ding, J. J., \& Chen, Q. S. (2011). MicroRNAs involved in the pathogenesis of phytophthora root rot of soybean (Glycine max). Agricultural Sciences in China, 10(8), 11591167.

77. Wang, M., Wang, Q., \& Wang, B. (2012). Identification and characterization of microRNAs in Asiatic cotton (Gossypium arboreum L.). PLoS One, 7(4), e33696.

78. Zhang, Q. (2007). Strategies for developing green super rice. Proceedings of the national Academy of Sciences, 104(42), 16402-16409.

79. Barozai, M. Y. K., Irfan, M., Yousaf, R., Ali, I., Qaisar, U., Maqbool, A., ... \& Riazuddin, S. (2008). Identification of micro-RNAs in cotton. Plant Physiology and Biochemistry, 46(89), 739-751.

80. Abdurakhmonov, I. Y., Devor, E. J., Buriev, Z. T., Huang, L., Makamov, A., Shermatov, S. E., ... \& Abdukarimov, A. (2008). Small RNA regulation of ovule development in the cotton plant, G. hirsutumL. BMC plant biology, 8(1), 93.

81. Yin, Z., Li, Y., Yu, J., Liu, Y., Li, C., Han, X., \& Shen, F. (2012). Difference in miRNA expression profiles between two cotton cultivars with distinct salt sensitivity. Molecular biology reports, 39(4), 4961-4970.

82. He, Q., Zhu, S., \& Zhang, B. (2014). MicroRNAtarget gene responses to lead-induced stress in cotton (Gossypium hirsutum L.). Functional \& integrative genomics, 14(3), 507-515.

83. Zhang, Y., Wang, W., Chen, J., Liu, J., Xia, M., \& Shen, F. (2015). Identification of miRNAs and their targets in cotton inoculated with Verticillium dahliae by high-throughput sequencing and degradome analysis. International Journal of Molecular Sciences, 16(7), 14749-14768.

84. Gao, P., Bai, X., Yang, L., Lv, D., Li, Y., Cai, H., ... \& Zhu, Y. (2010). Over-expression of osaMIR396c decreases salt and alkali stress tolerance. Planta, 231(5), 991-1001.

85. Lu, Y., Feng, Z., Bian, L., Xie, H., \& Liang, J. (2011). miR398 regulation in rice of the responses to abiotic and biotic stresses depends on CSD1 and CSD2 expression. Functional Plant Biology, 38(1), 44-53.

86. Yang, C., Li, D., Mao, D., Liu, X. U. E., Ji, C., Li, X., ... \& Zhu, L. (2013). Overexpression of micro RNA 319 impacts leaf morphogenesis and leads to enhanced cold tolerance in rice $(\mathrm{O}$ ryza sativa L.). Plant, cell \& environment, 36(12), 2207-2218.

87. Navarro, L., Dunoyer, P., Jay, F., Arnold, B., Dharmasiri, N., Estelle, M., ... \& Jones, J. D. (2006). A plant miRNA contributes to antibacterial resistance by repressing auxin signaling. Science, 312(5772), 436-439.

88. Li, Y., Zhang, Q., Zhang, J., Wu, L., Qi, Y., \& Zhou, J. M. (2010). Identification of microRNAs involved in pathogen-associated molecular pattern-triggered plant innate immunity. Plant physiology, 152(4), 2222-2231.

89. Lauter, N., Kampani, A., Carlson, S., Goebel, M., \& Moose, S. P. (2005). microRNA172 down- 
regulates glossy15 to promote vegetative phase change in maize. Proceedings of the National Academy of Sciences, 102(26), 9412-9417.

90. Chuck, G., Cigan, A. M., Saeteurn, K., \& Hake, S. (2007). The heterochronic maize mutant Corngrass 1 results from overexpression of a tandem microRNA. Nature genetics, 39(4), 544549.

91. Yang, Z., Wang, Y., Wei, X., Zhao, X., Wang, B., $\&$ Sui, N. (2017). Transcription profiles of genes related to hormonal regulations under salt stress in sweet sorghum. Plant molecular biology reporter, 35(6), 586-599.

92. Chang, H., Yi, B., Ma, R., Zhang, X., Zhao, H., \& Xi, Y. (2016). CRISPR/cas9, a novel genomic tool to knock down microRNA in vitro and in vivo. Scientific reports, 6(1), 1-12.
93. Schiml, S., \& Puchta, H. (2016). Revolutionizing plant biology: multiple ways of genome engineering by CRISPR/Cas. Plant methods, 12(1), 1-9.

94. Xie, K., \& Yang, Y. (2013). RNA-guided genome editing in plants using a CRISPR-Cas system. Molecular plant, 6(6), 1975-1983.

95. Jacobs, T. B., LaFayette, P. R., Schmitz, R. J., \& Parrott, W. A. (2015). Targeted genome modifications in soybean with CRISPR/Cas9. BMC biotechnology, 15(1), 1-10.

96. Basak, J., \& Nithin, C. (2015). Targeting noncoding RNAs in plants with the CRISPR-Cas technology is a challenge yet worth accepting. Frontiers in plant science, 6, 1001. 